\title{
Occurrence of Invasive Plant in three phytogeographical region of Bilaspur district of Chhattisgarh
}

Darakhsha Kauser Sheikh and Ashwini Kumar Dixit*

Department of Botany, Guru Ghasidas Vishwavidyalaya, India.

Received: 2017-11-07; Revised: 2017-11-09; Accepted: 2017-11-25

\begin{abstract}
The present study deals with comprehensive list of invasive species in the flora of Bilaspur district of Chhattisgarh, India with background information of family, nativity, mode of introduction, flowering and fruiting. 83 species of plant belong to 26 families were documented as invasive plant in all three phytogeographical regions (agricultural land, urban land and protected land). Both native and invasive plants were found in the same region, but the occurrence of invasive species were found high in the urban land (34.58\%) in comparison with protected land $(29.16 \%)$ and agriculture land (25\%). Habit wise analysis of invasive species showed $84.52 \%$ herbs, followed by $13.09 \%$ shrubs and $2.38 \%$ climbers. Maximum proportion of invasive plant are native to Tropical America and belongs to family Asteraceae (16), followed by Malvaceae (9), Euphorbiaceae (8), Fabaceae (6), Solanaceae (5), Convolvulaceae (4) and Lamiaceae (4).
\end{abstract}

Keywords: Occurrence, Nativity, Invasive species, Mode of Introduction.

\section{Introduction}

International Union for Conservation of Nature and Natural Resources (IUCN) defines invasive species as an alien species which established in natural or semi-natural ecosystems or habitat and threatens to native biological diversity. A large number of invasive species worldwide were introduced accidentally through transportation or intentionally for commercial purposes, value to human health and economic purposes (Ewel et al., 1999). Biological invasion of invading species cause species extinction (D'Antonio and Vitousek, 1992), and long-lasting change on habitats, which makes the restoration of native plants impossible, even when the invasive plants have been detached. Invasive plants affect the ecosystem functions, ecosystem processes, primary productivity, hydrology, geomorphology and biogeochemical cycle of an area (Vitousek et al., 1997; Gordon, 1998; Mack et al., 2000; Ehrenfeld, 2003; Liao et al., 2007). These species do not separate native plant diversity but compete with the local community for the limited natural resources and alter the rule of existence for all species (Vitousek et al., 1997). Litter of these invasive plants decomposed very slowly than those of the native plant community (Allison and Vitousek, 2004; Rothstein et al., 2004).

Mantri et al., (2002) gave 10 laws for examining the potential of invasive species. Aforesaid 10 laws are as follows (1) High input of viable propagules (2) High rate of aerial biomass production (3) Seeds or other reproductive unit with extensive period of dormancy (4) Adaptative features for long distance dispersal (5) Allelopathic effect on native plant community (6) Successful colonizer in disturbed

\section{Corresponding Author:}

Dr. Ashwini Kumar Dixit,

Associate Professor, Department of Botany,

Guru Ghasidas Vishwavidyalaya, India.

E-mail:dixitak@live.com land (7) Short time to overcome the reproductive maturity (8) Dense foliage canopy (9) Alternative mode of reproduction that facilitate to grow on adverse conditions (10) Wide distribution of species in different ecotypes (Figure-1). Long distance dispersal of seed results due to their adhesive properties (Cousens and Mortimer, 1995). Many plant seeds modified into special structure like awned, hooked, sticky, or barbed appendages for dispersal. These seeds stick to animal fur, insects and human clothes for dispersal (Sorensen, 1986). Davies and Sheley, (2007) gave a conceptual framework for dispersal of invasive plants seeds through wind, water, and vehicle.

GISD (Global Invasive Species Database) reported 100 worst invasive species throughout the world (Lowe et al., 2000). Numerous works regarding the distribution of invasive plants done all over the world (Daehler and Carino, 2000; Zhu et al., 2007). Similarly, various studies have been done in different states of India such as Himanchal Pradesh (Dogra et al., 2009), North West Himalaya (Negi and Hajra, 2007), Rajasthan (Pandey and Parmar, 1994), Himalayan region (Sekar, 2012) and special work on India (Raghubanshi et al., 2005; Reddy, 2008; Dogra et al., 2009; Nayar, 1977). Phytochemical screening of several invasive species has been analyzed by Baral, (2011); Rashmi and Rajkumar, (2011). Harmful effects of invasive plants such as habitat destruction, environmental damage, soil erosion etc. have been reported by Towns et al., 2006; and Jordon et al., 2008. Dixit and Chourasia, (2015) studied the ethnomedicinal importance of weeds of GGU campus, Bilaspur, CG. In 
Chhattisgarh state the knowledge of invasive plant is very poorly documented. So, the present paper focuses on the comprehensive list of invasive plant biodiversity in three phytogeographical region of Bilaspur District of Chhattisgarh.

\section{Materials and Methods}

To record the maximum number of plants random samplings were done during 2014 - 2016 in margins of study sites i.e. wastelands, forest cover area, river bank, agricultural field, bare lands, gardens etc. in district of Bilaspur C.G. These collected plants were categorized in life forms (herb, shrub and climber). All invasive plants were enumerated in alphabetic order followed with family, criteria of invasive species, nativity, flowering, fruiting and mode of introduction. Nativity of plants were checked through various literatures (Matthew, 1969; Maheswari and Paul, 1975; Saxena, 1991). Criteria of invasive plant was studied through periodic observations of SC (Successful colonizer) SLC (Small Life Cycle), DFC (Dense Foliage Canopy), DSE (Distribution of Species in different Ecosystem), AF (Adaptive Features) and AMR (Alternative Mode of Reproduction). Plants have been collected in flowering and fruiting conditions and compressed for herbarium preparation. Collected plant samples were identified with the help of flora of Bilaspur District, M.P. (Murti and Panigrahi, 1999).

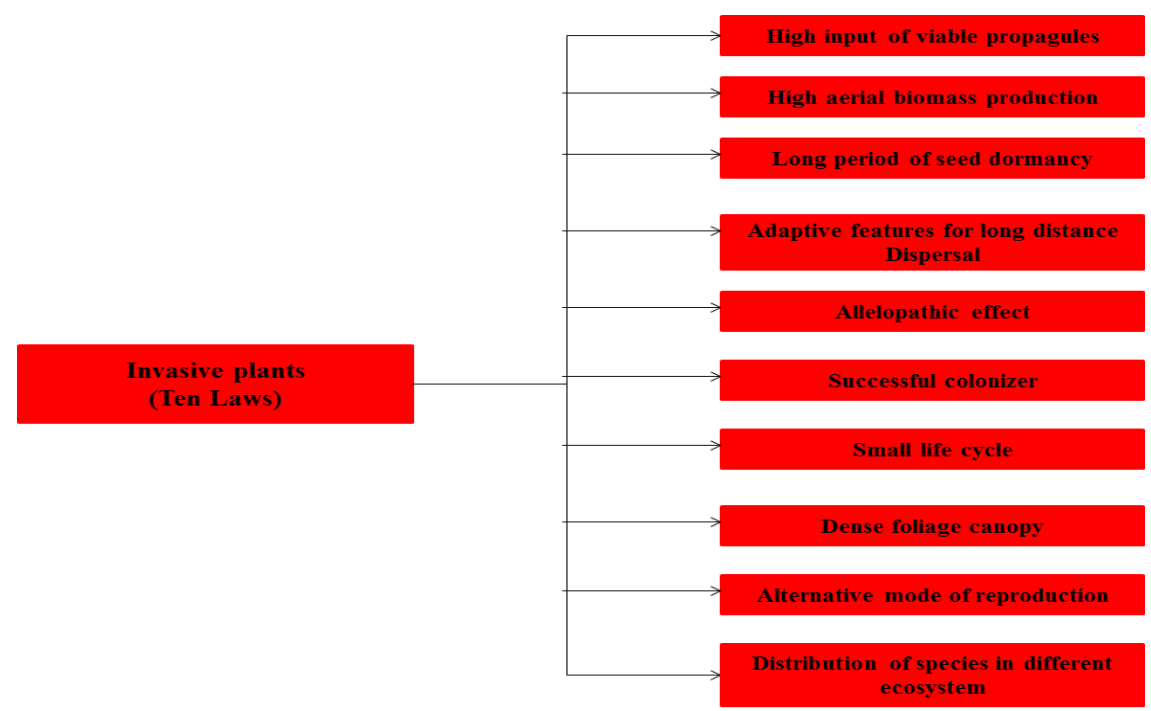

Figure 1. Ten laws criteria of invasive species. (Mantri et al., 2002)

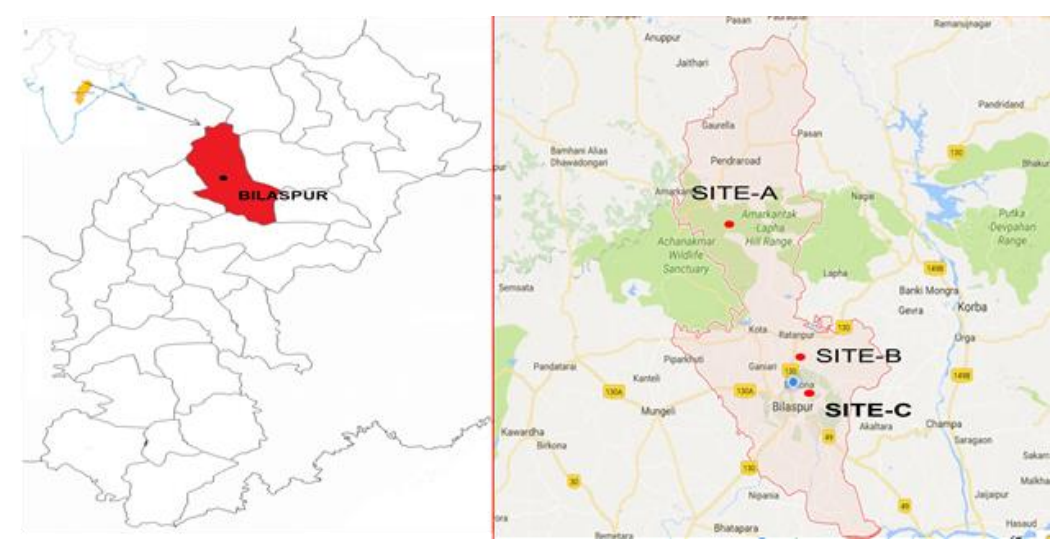

Figure 2. A map of study areas (red dots) in the position of Bilaspur district, Chhattisgarh, India; Site A Achanakmar Biosphere reserve (protected land); Site B - Turkadiah (Agriculture land); Site C - Bilaspur City (Urban area).

\section{Study Site}

Bilaspur district lies between $21^{\circ} 47^{\prime}$ and $23^{\circ} 8^{\prime}$ north latitudes and $81^{\circ} 14^{\prime}$ and $83^{\circ} 15^{\prime}$ east latitudes and is situated in the eastern part of Chhattisgarh state with geographical area of $6377 \mathrm{~km}^{2}$. It is divided into three phytogeographic regions; Protected land (Achanakmar Biosphere reserve), Agricultural land (Turkadiah) and Urban land (Bilaspur City). Climate of the region is typically tropical, subtropical and sub humid and dividable into three distinct seasons i.e. rainy (July to October) winter (November to Feb), and summer (March to June).

\section{Results}

Initially 240 plant species were observed and checked, out of which 84 plant species were found to be invasive. These plants were found along the 
roadside area, exposed slopes, roadways, gullies, besides track lines, crop fields and river banks throughout the study sites. (Table-1) A total of 84 invasive species belonging to 26 families were recorded from Bilaspur district of Chhattisgarh, out of which $84.52 \%$ species of herbs, $13.09 \%$ species of shrubs and $2.38 \%$ species of climbers (figure 2).

The most dominant families were Asteraceae (16), Malvaceae (9), Euphorbiaceae (8), Fabaceae (6), Solanaceae (5) Convolvulaceae (4), Lamiaceae (4), Acanthaceae (3), Amaranthaceae (3), Boraginaceae (3), Cleomaceae (3), Commelinaceae (2), Plantaginaceae (2), Poaceae (2) and Polygonaceae (2) (Figure-3). Eleven families of flowering plant represent one species each, i.e. Apocynaceae,
Balsaminaceae, Gentianaceae, Martyniaceae, Molluginaceae, Papaveraceae, Phyllanthaceae, Pontederiaceae, Rubiaceae, Verbenaceae and Vitaceae (Table -1).

Out of 84 invasive species, $34.58 \%$ were recorded in the urban land, $29.16 \%$ in the Agricultural land and $25 \%$ in the protected land (figure-4). In total of 84 invasive species, 80 species were introduced unintentionally, 2 ornamentally and 2 Agroforestry (table -1). Most of the invasive species were native to Tropical America (42), Tropical Africa (10), Asia (9), South America (5) Europe (3) and North America (Table-1). Very few species i.e. Merremia emarginata, Cayratia trifolia and Leucas cephalotes were native to Australia, Mediterranean and South Africa.

Table 1. List of invasive species of Bilaspur, Chhattisgarh, India.

\begin{tabular}{|c|c|c|c|c|c|c|c|}
\hline S.No. & Botanical name & Family & Habit & Criteria of IP species & $\begin{array}{c}\text { Mode of } \\
\text { Introduction }\end{array}$ & Nativity & $\begin{array}{l}\text { Flowering \& } \\
\text { Fruiting }\end{array}$ \\
\hline 1. & Abutilon birtum (Lam.) Sweet & Malvaceae & S & SC, SLC, DFC, DSE & $\mathrm{Ui}$ & Madagascar & March-Sep \\
\hline 2. & Acalypha indica $\mathrm{L}$. & Euphorbiaceae & $\mathrm{H}$ & SC, SLC, DFC, DSE & $\mathrm{Ui}$ & Asia & July- Oct \\
\hline 3. & Aerva lanata (L) Juss. Ex. Schult & Amaranthaceae & $\mathrm{H}$ & SC, SLC, DFC, DSE & $\mathrm{Ui}$ & Madagascar & Aug -Nov \\
\hline 4. & Aeschynomene indica Sensee auct, pp. & Fabaceae & $\mathrm{H}$ & SC, SLC, DFC, DSE & $\mathrm{Ui}$ & Trop. America & Aug - Jan \\
\hline 5. & Ageratum conyzoides $\mathrm{L}$. & Asteraceae & $\mathrm{H}$ & SC, SLC, DFC, DSE, AF & $\mathrm{O}$ & Trop. America & July - Jan \\
\hline 6. & Alternanthera sessilis (L) R.Br. ex DC. & Amaranthaceae & $\mathrm{H}$ & SC, SLC, DFC, DSE & $\mathrm{Ui}$ & Trop. America & July - Jan \\
\hline 7. & Antigonon leptopus Hook \& Arn & Polygonaceae & $\mathrm{C}$ & SC, SLC, DFC, DSE & $\mathrm{Ui}$ & Trop. America & March-Dec \\
\hline 8. & Argemone mexicana L. & Papaveraceae & $\mathrm{H}$ & SC, SLC, DFC, DSE & $\mathrm{Ui}$ & South America & Sep - Jan \\
\hline 9. & Bidens bipinnata $\mathrm{L}$. & Asteraceae & $\mathrm{H}$ & SC, SLC, DFC, DSE, AF & $\mathrm{Ui}$ & Trop. America & Aug-Oct \\
\hline 10. & Bidens pilosa L. & Asteraceae & $\mathrm{H}$ & SC, SLC, DFC, DSE, AF & $\mathrm{Ui}$ & Trop. America & July - Dec \\
\hline 11. & Blumea fistulosa (Roxb.) Kuntze & Asteraceae & $\mathrm{H}$ & SC, SLC, DFC, DSE, AF & $\mathrm{Ui}$ & Madagascar & Oct - March \\
\hline 12. & Blumea lacera (Burm.f.) DC. & Asteraceae & $\mathrm{H}$ & SC, SLC, DFC, DSE, AF & $\mathrm{Ui}$ & Trop. America & Aug - Feb \\
\hline 13. & Calotropis gigantea (L.) W.T. Aiton & Apocynaceae & S & SC, SLC, DFC, DSE, AF & $\mathrm{Ui}$ & Trop. Africa & $\begin{array}{l}\text { Throughout } \\
\text { the year }\end{array}$ \\
\hline 14. & Cassia alata $\mathrm{L}$. & Fabaceae & $\mathrm{S}$ & SC, SLC, DFC, DSE & $\mathrm{O}$ & West Indies & May - Dec \\
\hline 15. & Cassia occidentalis L. & Fabaceae & $\mathrm{H}$ & SC, SLC, DFC, DSE & $\mathrm{Ui}$ & South America & July - Dec \\
\hline 16. & Cassia tora $\mathrm{L}$. & Fabaceae & $\mathrm{H}$ & SC, SLC, DFC, DSE & $\mathrm{Ui}$ & South America & Aug - Dec \\
\hline 17. & Cayratia trifolia (L.) Domin & Vitaceae & $\mathrm{C}$ & SC, SLC, DFC, DSE & $\mathrm{Ui}$ & Mediterranean & June - Sep \\
\hline 18. & Celosia argentea $\mathrm{L}$. & Amaranthaceae & $\mathrm{H}$ & SC, SLC, DFC, DSE & $\mathrm{Fd}$ & Trop. Africa & Sep - Dec \\
\hline 19. & Cenchrus ciliaris $\mathrm{L}$. & Poaceae & $\mathrm{H}$ & SC, SLC, DFC, DSE & Fo & Trop. Africa & Sep - March \\
\hline 20. & Cbromolaena odorata (L.) R. M. King \& H. Rob. & Asteraceae & $\mathrm{S}$ & SC, SLC, DFC, DSE, AF & $\mathrm{Ui}$ & Trop. America & Dec-may \\
\hline 21. & $\begin{array}{l}\text { Chrozophora rottleri (Geiseler) A. Juss. Ex } \\
\text { Spreng. }\end{array}$ & Euphorbiaceae & $\mathrm{H}$ & SC, SLC, DFC, DSE & $\mathrm{Ui}$ & Trop. Africa & Sep- Feb \\
\hline 22. & Cleome gynandra $\mathrm{L}$ & Cleomaceae & $\mathrm{H}$ & SC, SLC, DFC, DSE & $\mathrm{Ui}$ & Trop. Africa & Sep - Dec \\
\hline 23. & Cleome rutidosperma DC. & Cleomaceae & $\mathrm{H}$ & SC, SLC, DFC, DSE & $\mathrm{Ui}$ & Trop. America & Aug - Dec \\
\hline 24. & Cleome viscosa $\mathrm{L}$. & Cleomaceae & $\mathrm{H}$ & SC, SLC, DFC, DSE & $\mathrm{Ui}$ & Trop. America & Aug - Dec \\
\hline 25. & Coldenia procumbens $\mathrm{L}$. & Boraginaceae & $\mathrm{H}$ & SC, SLC, DFC, DSE & $\mathrm{Ui}$ & Asia & Oct-March \\
\hline 26. & Commelina benghalensis $\mathrm{L}$. & Commelinaceae & $\mathrm{H}$ & SC, SLC, DFC, DSE & $\mathrm{Ui}$ & Asia & June - Dec \\
\hline 27. & Convolvulus nummularius $\mathrm{L}$. & Convolvulaceae & $\mathrm{H}$ & SC, SLC, DFC, DSE & $\mathrm{Ui}$ & Trop. America & October - Feb \\
\hline 28. & Corchorus fascicularis Lam. & Malvaceae & $\mathrm{H}$ & SC, SLC, DFC, DSE & $\mathrm{Ui}$ & Trop. America & July - Dec \\
\hline 29. & Crassocephalum crepidioides (Benth.) S. Moore & Asteraceae & $\mathrm{H}$ & SC, SLC, DFC, DSE, AF & $\mathrm{Ui}$ & Trop. America & July - Dec \\
\hline 30. & Croton bonplandianum Baill & Euphorbiaceae & $\mathrm{H}$ & SC, SLC, DFC, DSE, AF & $\mathrm{Ui}$ & South America & Aug - Dec \\
\hline 31. & Cynoglossum lanceolatum forsskal & Boraginaceae & $\mathrm{H}$ & SC, SLC, DFC, DSE, AF & $\mathrm{Ui}$ & Europe & July -Nov \\
\hline 32. & Datura alba Nees. & Solanaceae & $\mathrm{H}$ & SC, SLC, DFC, DSE & $\mathrm{Ui}$ & Trop. America & July - Feb \\
\hline 33. & Datura metel L. & Solanaceae & $\mathrm{H}$ & SC, SLC, DFC, DSE & $\mathrm{Ui}$ & Trop. America & Aug - Feb \\
\hline 34. & Eclipta prostrata (L.) & Asteraceae & $\mathrm{H}$ & SC, SLC, DFC, DSE, AF & $\mathrm{Ui}$ & Trop. America & June - March \\
\hline 35. & Eichbornia crassipes (C. Martius) Solms-Loub. & Pontederiácea & $\mathrm{H}$ & SC, SLC, DFC, DSE, AMR & $\mathrm{O}$ & Trop. America & Aug - Dec \\
\hline 36. & Elephantopus scaber $\mathrm{L}$. & Asteraceae & $\mathrm{H}$ & SC, SLC, DFC, DSE, AF & $\mathrm{Ui}$ & Asia & Aug-Dec \\
\hline 37. & Emilia Sonchifolia (L.) Dc. Ex. Wight & Asteraceae & $\mathrm{H}$ & SC, SLC, DFC, DSE, AF & $\mathrm{Ui}$ & Trop. America & Aug -March \\
\hline 38. & Euphorbia beterophylla $\mathrm{L}$. & Euphorbiaceae & $\mathrm{H}$ & SC, SLC, DFC, DSE & $\mathrm{Ui}$ & Trop. America & Aug - Dec \\
\hline 39. & Euphorbia birta L. & Euphorbiaceae & $\mathrm{H}$ & SC, SLC, DFC, DSE & $\mathrm{Ui}$ & Trop. America & Aug-Dec \\
\hline 40. & Euphorbia thymifolia $\mathrm{L}$. & Euphorbiaceae & $\mathrm{H}$ & SC, SLC, DFC, DSE & $\mathrm{Ui}$ & Trop. America & June- Oct \\
\hline 41. & Exacum pedunculatum $\mathrm{L}$. & Gentianaceae & $\mathrm{H}$ & SC, SLC, DFC, DSE & $\mathrm{Ui}$ & Mexico & Feb- April \\
\hline 42. & Grangea maderaspatana (L.) Poir. & Asteraceae & $\mathrm{H}$ & SC, SLC, DFC, DSE, AF & $\mathrm{Ui}$ & South America & Aug - Dec \\
\hline 43. & Heliotropium indicum $\mathrm{L}$. & Boraginaceae & $\mathrm{H}$ & SC, SLC, DFC, DSE & $\mathrm{Ui}$ & Asia & Feb-June \\
\hline 44. & Hygrophila auriculata (Schumach.) Heine & Acanthaceae & $\mathrm{H}$ & SC, SLC, DFC, DSE & $\mathrm{Ui}$ & Asia & June- Dec \\
\hline 45. & Hyptis suaveolens (L.) Poit & Lamiaceae & $\mathrm{H}$ & SC, SLC, DFC, DSE & $\mathrm{Ui}$ & Trop. America & Aug - March \\
\hline 46. & Impatiens balsamina $\mathrm{L}$. & Balsaminaceae & $\mathrm{H}$ & SC, SLC, DFC, DSE & $\mathrm{O}$ & Trop. America & July - Jan \\
\hline 47. & Indigofera linnaei Ali. & Fabaceae & $\mathrm{H}$ & SC, SLC, DFC, DSE & $\mathrm{Ui}$ & Trop. Africa & Aug - Jan \\
\hline 48. & Ipomoea carnea Jace. & Convolvulaceae & $\mathrm{H}$ & $\begin{array}{l}\text { SC, SLC, DFC, DSE, AF, } \\
\text { AMR }\end{array}$ & $\mathrm{Ui}$ & Trop. America & $\begin{array}{l}\text { Throughout } \\
\text { the year }\end{array}$ \\
\hline 49. & Ipomoea bederifolia $\mathrm{L}$. & Convolvulaceae & $\mathrm{H}$ & SC, SLC, DFC, DSE & $\mathrm{Ui}$ & Trop. America & Aug - Dec \\
\hline 50. & Jatropha curcas $\mathrm{L}$. & Euphorbiaceae & S & SC, SLC, DFC, DSE & Af & Trop. America & June-Oct \\
\hline 51. & Jatropha gossypifolia L. & Euphorbiaceae & S & SC, SLC, DFC, DSE & Af & Mexico & July- Nov \\
\hline 52. & Lagascea mollis Cav. & Asteraceae & $\mathrm{H}$ & SC, SLC, DFC, DSE & $\mathrm{Ui}$ & Central America & Aug - Feb \\
\hline
\end{tabular}




\begin{tabular}{|c|c|c|c|c|c|c|c|}
\hline 53. & Lantana camara $\mathrm{L}$. & Verbenaceae & $\mathrm{S}$ & SC, SLC, DFC, DSE & $\mathrm{O}$ & Trop. America & $\begin{array}{l}\text { Throughout } \\
\text { the year }\end{array}$ \\
\hline 54. & Leonotis nepetifolia (L.) R. Br. & Lamiaceae & $\mathrm{H}$ & SC, SLC, DFC, DSE & $\mathrm{Ui}$ & Trop. Africa & Aug-Dec \\
\hline 55. & Leucas cephalotes (Roth) Spreng & Lamiaceae & $\mathrm{H}$ & SC, SLC, DFC, DSE & $\mathrm{Ui}$ & South Africa & Aug - Dec \\
\hline 56. & Malacbra capitata (L.) L. & Malvaceae & $\mathrm{H}$ & SC, SLC, DFC, DSE & $\mathrm{Ui}$ & Trop. America & June-Jan \\
\hline 57. & Malvastrum coromandelianum (L.) Garcke & Malvaceae & $\mathrm{H}$ & SC, SLC, DFC, DSE & $\mathrm{Ui}$ & Trop. America & Aug - Jan \\
\hline 58. & Martynia annua $\mathrm{L}$. & Martyniaceae & $\mathrm{S}$ & SC, SLC, DFC, DSE, AF & $\mathrm{Ui}$ & Trop. America & Aug - Dec \\
\hline 59. & Mecardonia procumbens (Mill.) Small & Plantaginaceae & $\mathrm{H}$ & SC, SLC, DFC, DSE & $\mathrm{Ui}$ & Asia & Aug - Jan \\
\hline 60. & Melocbia corchorifolia $\mathrm{L}$. & Malvaceae & $\mathrm{H}$ & SC, SLC, DFC, DSE & $\mathrm{Ui}$ & Trop. America & Aug - Dec \\
\hline 61. & Merremia emarginata (Burm. fil.) Hall. fil. & Convolvulaceae & $\mathrm{H}$ & SC, SLC, DFC, DSE & $\mathrm{Ui}$ & Australia & Nov - Feb \\
\hline 62. & Mollugo pentaphylla L. & Molluginaceae & $\mathrm{H}$ & SC, SLC, DFC, DSE & $\mathrm{Ui}$ & Trop. Africa & June-Sep \\
\hline 63. & Murdannia nudiflora (L.) Brenn & Commelinaceae & $\mathrm{H}$ & SC, SLC, DFC, DSE & $\mathrm{Ui}$ & Trop. Africa & June-Aug \\
\hline 64. & Parthenium hysterophorus $\mathrm{L}$. & Asteraceae & $\mathrm{H}$ & SC, SLC, DFC, DSE, AF & $\mathrm{Ui}$ & North America & July-Feb \\
\hline 65. & Peristrophe paniculata (Forssk.) R.K. Brummitt & Acanthaceae & $\mathrm{H}$ & SC, SLC, DFC, DSE & $\mathrm{Ui}$ & Trop. America & Aug - Jan \\
\hline 66. & Phyllanthus virgatus $\mathrm{G}$. Forst & Phyllanthaceae & $\mathrm{H}$ & SC, SLC, DFC, DSE & $\mathrm{Ui}$ & Europe & Sep - Dec \\
\hline 67. & Physalis minima $\mathrm{L}$. & Solanaceae & $\mathrm{H}$ & SC, SLC, DFC, DSE & Af & Trop. America & July - Jan \\
\hline 68. & Plectranthus mollis (W.T.) Aiton) Spreng & Lamiaceae & $\mathrm{H}$ & SC, SLC, DFC, DSE & $\mathrm{Ui}$ & Trop. America & Aug - Dec \\
\hline 69. & Ruellia tuberosa $\mathrm{L}$. & Acanthaceae & $\mathrm{H}$ & SC, SLC, DFC, DSE & $\mathrm{O}$ & Trop. America & Aug - Dec \\
\hline 70. & Rumex crispus $\mathrm{L}$. & Polygonaceae & $\mathrm{H}$ & SC, SLC, DFC, DSE & $\mathrm{Ui}$ & Europe & Oct - Feb \\
\hline 71. & Scoparia dulcis L. & Plantaginaceae & $\mathrm{H}$ & SC, SLC, DFC, DSE & $\mathrm{Ui}$ & Trop. America & July - Dec \\
\hline 72. & Setaria viridis (L.) P. Beauv. & Poaceae & $\mathrm{H}$ & SC, SLC, DFC, DSE, AF & Af & Trop. America & June-Sep \\
\hline 73. & Sida acuta Burm.f. & Malvaceae & $\mathrm{H}$ & SC, SLC, DFC, DSE, AF & $\mathrm{Ui}$ & Trop. America & July - Dec \\
\hline 74. & Sida cordata (Burm.f.) Borss. Waalk & Malvaceae & $\mathrm{H}$ & SC, SLC, DFC, DSE & $\mathrm{Ui}$ & Asia & Aug-Dec \\
\hline 75. & Solanum sisymbrifolium Lam. & Solanaceae & $\mathrm{S}$ & SC, SLC, DFC, DSE & $\mathrm{Ui}$ & Trop. America & Aug - Feb \\
\hline 76. & Solanum torvum $\mathrm{Sw}$. & Solanaceae & $\mathrm{S}$ & SC, SLC, DFC, DSE & $\mathrm{Ui}$ & West Indies & Oct - March \\
\hline 77. & Spermooce hispida L. & Rubiaceae & $\mathrm{H}$ & SC, SLC, DFC, DSE & $\mathrm{Ui}$ & Trop. America & June - Oct \\
\hline 78. & Spilanthes acmella (L.) L. & Asteraceae & $\mathrm{H}$ & SC, SLC, DFC, DSE & $\mathrm{Ui}$ & North America & Oct - March \\
\hline 79. & Tephrosia purpurea (L.) Pers. & Fabaceae & $\mathrm{H}$ & SC, SLC, DFC, DSE & $\mathrm{Ui}$ & Asia & June - Oct \\
\hline 80. & Tridax procumbens L. & Asteraceae & $\mathrm{H}$ & SC, SLC, DFC, DSE, AF & $\mathrm{Ui}$ & Central America & $\begin{array}{l}\text { Throughout } \\
\text { the year }\end{array}$ \\
\hline 81. & Triumfetta rhomboidea jacq. & Malvaceae & $\mathrm{H}$ & SC, SLC, DFC, DSE, AF & $\mathrm{Ui}$ & Trop. America & June - Dec \\
\hline 82. & Urena lobata L. & Malvaceae & $\mathrm{H}$ & SC, SLC, DFC, DSE, AF & $\mathrm{Ui}$ & Trop. Africa & July - Dec \\
\hline 83. & Xanthium strumarium L. & Asteraceae & $\mathrm{H}$ & SC, SLC, DFC, DSE, AF & $\mathrm{Ui}$ & Trop. America & Aug - March \\
\hline
\end{tabular}
Life form: H-Herb; C-Climber; S-Shrub; Criteria of invasive plants: SC (Successful colonizer) SLC (Small Life Cycle), DFC (Dense Foliage Canopy), DSE (Distribution of Species in different Ecosystem), AF (Adaptive Features), AMR (Alternative Mode of Reproduction); Mode of introduction: Ui - Unintentional, Fd- Fodder, O- Ornamental, Af -Agroforestry.

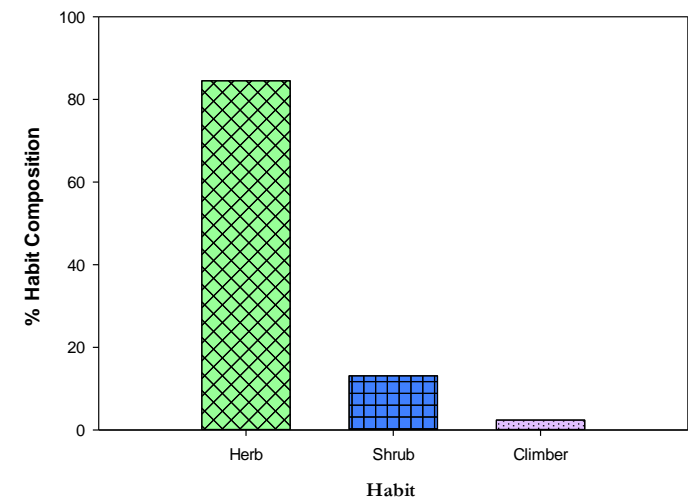

Figure 2. Percent composition of habit analysis of invasive plant.

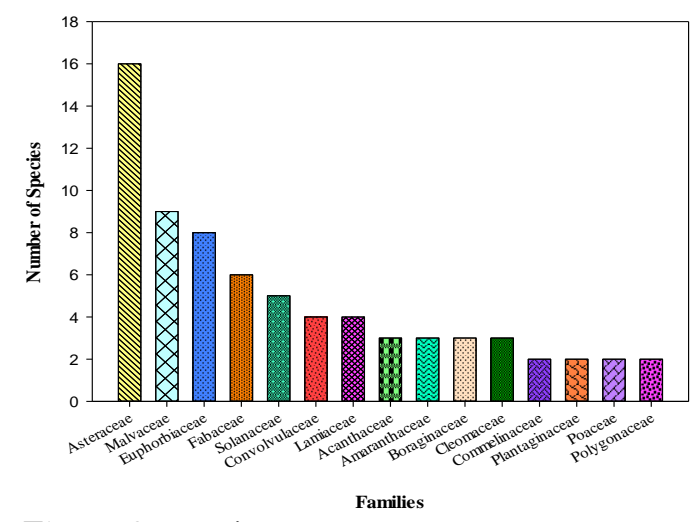

Figure 3. Dominant family graph of invasive plant.

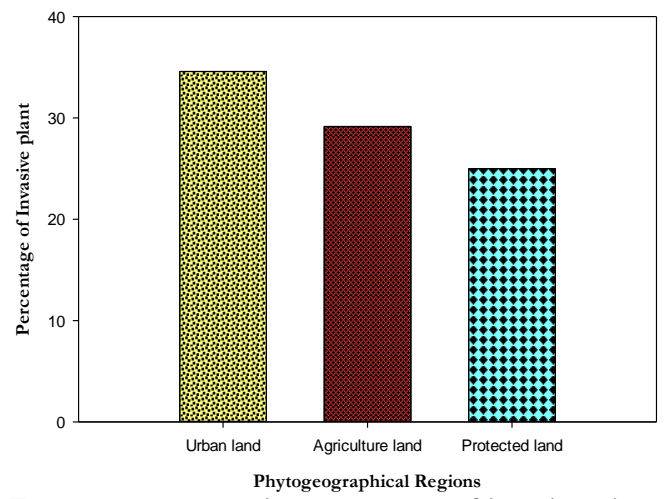

Figure 4. Percent wise occurrence of invasive plants in all three phytogeographical regions.

\section{Discussion}

This work shows that maximum number of invasive plants were found in urban land, some in agriculture land and least in protected land. Establishment of invasive plant species in urban lands depends on human disturbance (Pyšek et al, 1998 and Byers 2002). Dominant family of the species belongs to Asteraceae family whose seeds possess adaptive features for dispersals. In Spite of Eichhornia crassipes and Ipomoea carnea all other species were reproduced by seeds but the above aforesaid species are reproduced through seeds and propagative stem. $84.52 \%$ of invasive plants were herb which have small life cycles and occur specifically in rainy and winter season, remaining $13.09 \%$ species of shrub have long life cycles and present throughout the year. 

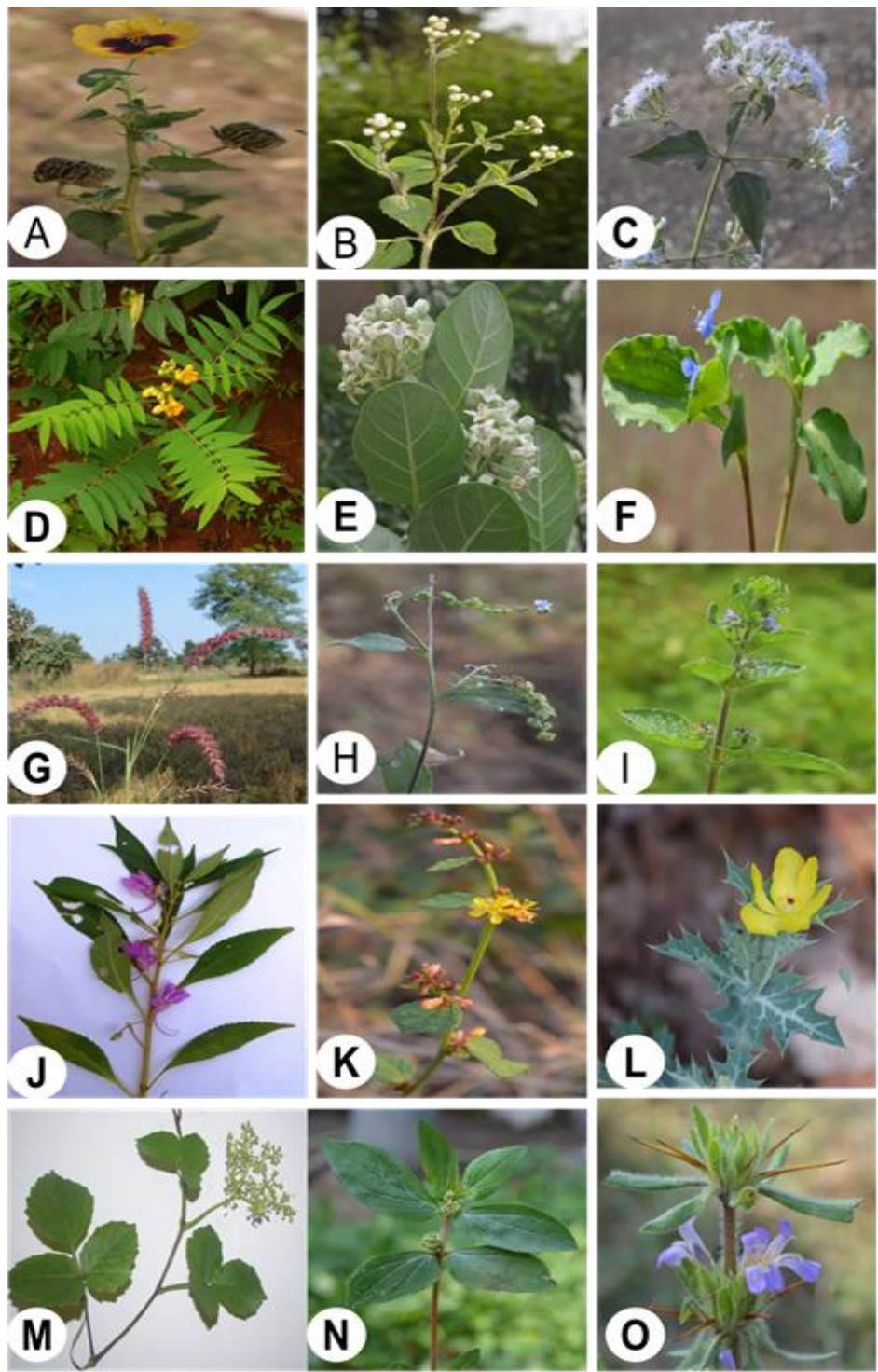

Plate 1: (A-O);
(A) Abutilon birtum
(D) Cassia occidentalis
(G) Cenchrus ciliaris
(J) Impatiens balsamina
(M) Cayratia trifolia

(B) Ageratum conyzoides

(E) Calotropis gigantea

(H) Cynoglossum lanceolatum

(C) Chromolaena odorata

(F) Commelina benghalensis

(I) Hyptis suaveolens

$\begin{array}{ll}\text { (K) Triumfetta rhomboidea } & \text { (L) Argemone mexicana }\end{array}$

(N) Euphorbia birta

(O) Hygrophila auriculata

\section{Conclusion}

This work focuses heavily on presence of invasive species in all three phytogeographical areas of Bilaspur district in Chhattisgarh. It is a first attempt to prepare a checklist of invasive plant species which will help to understand the presence, distribution and reproduction of plant in Bilaspur district of Chhattisgarh. Present work will provide a better way to plan for early detection of invasion and establishment of routine monitoring of invasive plant by land managers, taxonomist, agriculturist, botanist and ecologist. One of the advantages of this work is prevention of certain diseases like asthma, allergy etc. caused by invasive plant and save loss of native species which have a great medical importance.

\section{Reference}

1. Allison, S.D. and P.M., Vitousek "Rapid nutrient cycling in leaf litter from invasive plants in Hawaii” Oecologia. 141.4 (2004): 612619.

2. Barel, B. "Antagonistic characteristics and phytochemical screening of invasive alien species of Nepal Himalaya” International Journal of Pharmaceutical \& Biological Archives 2.5 (2011): 1444- 1450.

3. Cousens, R. and Mortimer, M. "Dynamics of Weed Populations" New York: Cambridge University Press (1995) 332 p. 
4. D'Antonio, C. and P.M., Vitousek "Biological invasions by exotic grasses, the grass/fire cycle, and global change" Annual review of Ecology and systematics 23 (1992): 63-87.

5. Daehler, C.C. and Debbie, A.SC. "Predicting invasive plants: prospects for a general screening system based on current regional models" Biological Invasions 2 (2000): 93-102.

6. Davies, K.W. and Roger, S.L. "A conceptual framework for preventing the spatial dispersal of invasive plants" Weed Science 55.2 (2007):178-184.

7. Dixit, A.K. and Bhaskar, C. "Ethno-medicinal uses of weeds of Guru Ghasidas central university, Bilaspur, (CG) India" The Journal of Ethnobiology and Traditional Medicine Photon 125 (2005): 1046-1054.

8. Dogra, K.S., R.K., Kohli and S.K., Sood "An assessment and impact of three invasive species in the Shivalik hills of Himachal Pradesh, India" International Journal of Biodiversity and Conservation. 1.1 (2009): 004-010.

9. Ehrenfeld, JG. "Effects of exotic plant invasions on soil nutrient cycling processes" Ecosystems 6.6 (2003): 503 - 523

10. Ewel, J.J., Dennis, J.O., Joy, B., Curtis, C.D., Carla, M.D., Luis, D.G., Doria, R.G., Richard, J.H., Alan, H., Keith, R.H.C.E.H., Marcy, L.H., Roger, R.B.L., William, G.L., Lloyd, L.L., David, H.L., Svata, M.L., Ariel, E.L., Peter, B.M., David, M.R. and Peter, M.V. "Deliberate introductions of species: Research needs" BioScience 49.8 (1999):619-630.

11. Gordon, DR. "Effects of invasive, nonindigenous plant species on ecosystem processes: lessons from Florida" Ecological Applications 8.4 (1998): 975 - 989.

12. Jordon, N.R., Diane, L.L. and Sheri, C.H. "Soil modification by invasive plants: effect on native and invasive species of mixed- grass prairies" Biol invasions 10.2 (2008): 177-190.

13. Liao, C., Ronghao, P., Yiqi, L., Xuhui, Z., Xiaomen, W., Changming, F., Jiakuan, C. and Bo, L. "Altered ecosystem carbon and nitrogen cycles by plant invasion: a meta-analysis" New Phytologist. 177.3 (2007):706-714.

14. Lowe, S., M. Browne, S., Boudjelas and M.D., Poorter "100 of the World's Worst Invasive Alien Species: A selection from the global invasive species database" Published by The Invasive Species Specialist Group (ISSG) a specialist group of the Species Survival Commission (SSC) of the
World Conservation Union (IUCN) (2000) :12pp.

15. Mack, R.N., Daniel, S., W., Londsale, Harry, E., Michael, C., and Fakhri, A.B. "Biotic invasions: Causes, epidemiology, global consequences, and control" Ecological Applications 10.3 (2000): 689-710.

16. Maheswari, J.K., and S.R., Paul "The exotic flora of Ranchi" J Bombay Nat Hist Soc 72.1 (1975): $158-88$.

17. Mantri, A., Annapurana, C. and J.S., Singh "Terrestrial plant invasions and global change. In: Bioresources and environment, Tripathi, G. and Tripathi, Y.C. (eds) Campus books, New Delhi. (2002): 25-44.

18. Matthew, KM. Exotic flora of Kodaikanal and Palni hills. Rec Bot Surv India 20.1 (1969): 1 241.

19. Nayar, M.P. "Changing patterns of the Indian Flora" Bulletin of botanical survey of India. 19.1-4 (1977): 145-155.

20. Negi, P.S. and P.K., Hajra "Alien flora of Doon valley, Northwest Himalaya" Current Science 92.7 (2007): 968 - 978.

21. Pandey, R.P. and P.J. Parmar, "The exotic flora of Rajasthan" J Econ Tax Bot. 18.1 (1994): 105 21.

22. Panigrahi, G. and S.K., Murti, "Flora of Bilaspur District M.P." Published by Botanical Survey of India, Calcutta. (1989).

23. Raghubanshi, A.S., L.C., Rai, J.P. Gaur, and J.S. Singh, "Invasive alien species and biodiversity in India" Current Science. 88.4 (2005): 1-2.

24. Rashmi, S. and H.G., Rajkumar "Phytochemical Analysis and in vitro Evaluation of Antifungal Activity of Five Invasive Plant Species against Macrophomina Phaseolina (Tassi) Goid" International Journal of Plant Research. 1.1 (2011): 11-15.

25. Reddy, C.S. "Catalogue of invasive alien flora of India" Life science journal 5.2 (2008): 20-25.

26. Rothstein DE, Peter, M.V. and Breana, L.S. "An exotic tree alters decomposition and nutrient cycling in a Hawaiian montane forest" Ecosystems 7.8 (2004): $805-814$.

27. Saxena, KG. "Biological invasion in the Indian subcontinent: review of invasion by plants" In: Ecology of Biological Invasion in the Tropics (1991): $53-73$. 
28. Sekar, K.C. "Invasive alien plants of Indian Himalayan region diversity and implication'. American journal of plant sciences. 3 (2012): 177184.

29. Sorensen, A.E. "Seed dispersal by adhesion" Annu. Rev. Ecol. Syst. 17(1986): 443-463.

30. Towns, D.R. Ian, A.E.A. and Charles, H.D "Have the harmful effects of introduced rats on islands been exaggerated?" Biological invasion 8.4 (2006): 863-891.

31. Vitousek, P.M., Carla, M.D., Lloyd, L.L., Marcel, R. and Randy, W. "Introduced species: a significant component of human-caused global change" New Zealand Journal of Ecology 21 (1997): 1-16.

32. Zhu, L., O.J. Sun, Sang, W., Li, Z. and Ma, K. "Predicting the spatial distribution of an invasive plant species (Eupatorium adenophorum) in China" Landscape Ecol. 22 (2007): 1143-1145.

\section{Cite this article as:}

Darakhsha Kauser Sheikh and Ashwini Kumar Dixit. Biodiversity and Distribution of Invasive Plant in three Phytogeographical region of Bilaspur District of Chhattisgarh, Annals of Plant Sciences 6.12 (2017) pp. 1872-1878.

doi: http://dx.doi.org/10.21746/aps.2017.6.12.13

Source of support: Nil

Conflict of interest: $\mathrm{Ni}$ 